\title{
A depth-averaged two-dimensional sediment transport model for environmental studies in the Scheldt Estuary and tidal river network
}

\author{
Olivier Gourgue (1,2), Willy Baeyens (3), Margaret Chen (4), Anouk de Brauwere (1,2,3), Benjamin de Brye \\ $(1,2)$, Eric Deleersnijder (1,5), Marc Elskens (4), Vincent Legat $(1,2)$ \\ (1) Université catholique de Louvain (UCL), Institute of Mechanics, Materials and Civil engineering (iMMC), \\ Louvain-la-Neuve, Belgium, (2) Université catholique de Louvain (UCL), Georges Lemaître Centre for Earth and Climate \\ Research (TECLIM), Louvain-la-Neuve, Belgium, (3) Vrije Universiteit Brussel (VUB), Laboratory of Analytical and \\ Environmental Chemistry (ANCH), Brussels, Belgium, (4) Vrije Universiteit Brussel (VUB), Department of Hydrology and \\ Hydraulic Engineering (HYDR), Brussels, Belgium, (5) Université catholique de Louvain (UCL), Earth and Life Institute \\ (ELI), Georges Lemaître Centre for Earth and Climate Research (TECLIM), Louvain-la-Neuve, Belgium
}

We present the sediment module designed for the two-dimensional depth-averaged and one-dimensional sectionaveraged components of the finite-element model SLIM (Second-generation Louvain-la-Neuve Ice-ocean Model) in the framework of its application to the tidal part of the Scheldt Basin. This sediment transport module focuses on fine-grained, cohesive sediments. It is a necessary tool to undertake environmental biogeochemical studies, in which fine sediment dynamics play a crucial role.

The variables are the suspended sediment concentration (SSC) and the concentration of the sediments freshly deposited on the bottom. Sediment dynamics is controlled by the transport of SSC by advection and diffusion, while deposition and resuspension processes also depend on other physical, chemical and biological properties. Besides building a functioning fine sediment transport model, the aim of this study is to identify, parametrize and quantify the key processes that are necessary to represent satisfactorily the suspended sediment dynamics in the Scheldt Estuary and tidal river network.

The settling velocity of suspended sediments is influenced by flocculation. The important factors governing this process include the SSC itself, the turbulence, the shear stress, the salinity, the biological activity and some physicochemical properties (e.g. pH). In this study, only SSC, salinity and biological activity are explicitely taken into account. Moreover, the influence of the biological activity on the bottom layer erodibility is considered as well, along with the distribution of sediment types along the estuary, as the presence of sand increases the ability of the bottom layer to erode. Finally, the influence of a convergence zone between bottom currents carrying large amounts of fine sediments is also considered.

The computer cost of a two-dimensional model is significantly smaller than that of the three-dimensional models traditionally deemed indispensable in sediment transport modeling. Even if the present simplified model is rather designed for a specific situation, it produces results that are rather similar to those obtained with more complex, three-dimensional tools, but also at a lower cost. Therefore, it is believed that the model presented herein is suitable for long-term environmental simulations in the Scheldt Estuary, especially when limited computer resources are available. This is the main achievement of the present study. 